\title{
Effect of Different Implicit Social Networks on Recommending Research Papers
}

\author{
Shaikhah Alotaibi \\ University of Saskatchewan \\ 181 Thorvadlson Building \\ 110 Science Place \\ Saskatoon, SK, Canada \\ Shaikhah.otaibi@usask.ca
}

\begin{abstract}
Combining social network information with collaborative filtering recommendation algorithms has successfully reduced some of the drawbacks of collaborative filtering and increased the accuracy of recommendations. However, all approaches in the domain of research paper recommendation have used explicit social relations that users have initiated which has the problem of low recommendation coverage. We argued that the available data in social bookmarking Web sites such as CiteULike or Mendeley could be exploited to connect similar users using implicit social connections based on their bookmarking behavior. In this paper, we propose three different implicit social networks - readership, co-readership, and tag-basedand we compare the recommendation accuracy of several recommendation algorithms using data from the proposed social networks as input to the recommendation algorithms. Then, we test which implicit social network provides the best recommendation accuracy. We found that, for the most part, the social recommender is the best algorithm and that the readership network with reciprocal social relations provides the best information source for recommendations but with low coverage. However, the co-readership network provide good recommendation accuracy and better user coverage of recommendation.
\end{abstract}

\section{Keywords}

Social networks; hybrid recommendation; paper recommendation; social bookmarking Web sites; collaborative filtering.

\section{INTRODUCTION}

Scholarly papers both help to update researchers on new research in their areas of interest and serve as a directory of other researchers with similar interests with whom researchers can collaborate. However, as publishers, online journals, and conferences proliferate, the number of new published papers has become overwhelming. For this reason, many recommender systems (RSs) have been proposed to help readers in these tasks by suggesting a list of potential papers to users. The two main algorithms used by RSs are content-based filtering (CBF) and collaborative filtering $(\mathrm{CF}) . \mathrm{CBF}$ is based on information retrieval techniques that compare a paper's features (e.g., title, abstract, keywords, publication year) with the researchers' features (e.g., interests or previous search queries) to find matches [1]. In contrast, CF (e.g., [12]) uses the similarity of paper ratings to find users similar to the target user and recommend papers that these users have liked. Hybrid recommending approaches (e.g., [17]) use a combination of the $\mathrm{CBF}$ and $\mathrm{CF}$ approaches to alleviate the drawbacks of both approaches.

With the advent of social networks in applications such as social bookmarking systems (e.g., CiteULike, Mendeley), which researchers often use to manage their digital paper repositories and bookmark libraries, users can be connected through different social relations. A social bookmarking service provides many clues for interest similarities between users based on their behavior in the system and their publication authorship. Surprisingly, however, none of the

\author{
Julita Vassileva \\ University of Saskatchewan \\ 178 Thorvadlson Building \\ 110 Science Place \\ Saskatoon, SK, Canada \\ jiv@cs.usask.ca
}

popular social bookmarking tools have used the wealth of social data they store to build a social RS. However, there are some studies that incorporate the social information into $\mathrm{CF}$ techniques to increase the recommendation accuracy. Although social recommenders perform well, the social information should be available which is not guaranteed for all users. Thus, the social recommenders have lower user coverage [7]. User coverage of the recommendation is the ratio of users who receive nonempty recommended sets to the total number of users [2]. Previous studies also showed that there is a tradeoff between the recommendation accuracy and the coverage of the recommendation [4]. In this paper, We aim to analyze different resources for finding similar users and to test which resource and which recommender approach give the best balance between accuracy and coverage. Therefore, we propose three implicit social networks that exploit data from the users' publication list (if there is one) and bookmarked papers in the social bookmarking Web sites. Users need not enter more data in the system, which completely infers the data used to build the implicit social network.

We have organized the rest of the paper as follows. Section 2 briefly discusses related work. Section 3 describes the three proposed implicit social networks. Section 4 describes our experiment design and the dataset this paper uses, and section 5 explains the experiments and result analysis. Finally, section 6 discusses our conclusions and future work.

\section{RELATED WORK}

Social recommendation can be defined to be any recommender system that includes social relations as an extra input [16]. Thus, social recommenders are hybrid recommender systems that combine social relationships (e.g. membership, friendship, following relations) with another recommendation method, most commonly CF. Rather than using only the user-item matrix as the traditional $\mathrm{CF}$, a social recommendation mechanism uses two matrices: a user-item matrix, which represents the items that are rated by the user, and a user-user matrix, which represents the social relations between users. Many studies demonstrate that using social information in the recommendation process reduces the effect of the data sparsity and cold start problems [11] and enhances prediction accuracy [9].

There are many approaches combining CF recommender with a social network based on explicit social relations between users (e.g. [11, 13]). Explicit relationships are those that are initiated by users, for example, following on Twitter or CiteULike, being friends on Facebook, or in general connection that is made with the awareness or agreement of both users. For example, Massa and Avesani [11] propose a trust graph-based RS that uses trust values given by users in addition to similarity measures to reduce the data sparseness that affects new users.

Existing research has explored also the use of implicit networks in social recommender systems. Implicit social networks are constructed by inferring relationships between users that may not exist in the real world, and the users may be unaware of them. For example, the users 
that belong to the same neighbourhood in a CF could be considered as part of an implicit network constructed by relating users who gave similar ratings to the same items. These implicit relationships have been often called "trust" $[1,3,13]$.

However, very few studies incorporate social relations in the domain of research paper recommendations. For example, PubRec is an RS that suggests to the target user, for a particular paper of interest, the most related papers from the libraries of other users to whom that user is socially connected [14]. PReSA [15] takes advantage of the available data on social bookmarking Web sites (e.g., CiteULike), such as bookmarked papers, metadata, and users' connections, to recommend papers from the users' connections' libraries that are similar and popular among the users' social connections. Both PubRec and PReSA consider the explicit relationships among users in the recommendation process. Lee and Brusilovsky studied three explicit social networks - watching networks [5], group membership [6], and collaboration networks [7] - to find the extent of interest similarities between users involved in those networks and compare the recommendations watching networks produced to the recommendations traditional $\mathrm{CF}$ produced [5]. Their results showed that the watching network cannot compete with $\mathrm{CF}$, that the similarities between users' libraries in group membership networks are insignificant [6], and that the similarity between two users connected using co-authorship networks is comparable to user connections using explicit networks, which require agreement between the parties [7]. All of the above studies in recommending research papers depend on the existence of users' connections to make recommendations which is usually not available for all users. Thus, the number of users who can get recommendations is reduced.

\section{PROPOSED IMPLICIT SOCIAL NETWORKS}

Using data collected from CiteULike, we built three implicit social networks (ISNs) based on users' bookmarking behavior. CiteULike is a social bookmarking Web site for bookmarking research papers that has been in active use since November 2004; the site currently has $8,217,384$ bookmarked papers.

\subsection{Network 1: Readership Implicit Social Network}

The readership ISN connects users to the authors of the papers that they have bookmarked. We assume that if users bookmark specific papers, interest overlap exists between the bookmarkers and the authors of the papers; this overlap increases with the increase in the number of papers users bookmark from the same author. The relation could be unidirectional or reciprocal. The relation is unidirectional if only one of the users in this relation has bookmarked the other user's publications. The relation is reciprocal if both users have bookmarked each other's publications. Figure 1 shows the relations in this network, which are depicted as black arrows. For example, the relation between user 3 and user 5 is reciprocal, while the relation between user 3 and user 1 is unidirectional; user 3 is the paper's bookmarker and user 1 is the paper's author. The numbers on the arrows represent the strength of the relations. For example, the strength of the relation between user 3 and user 1 is five, which means that user 3's library contains five bookmarked papers authored by user 1 .

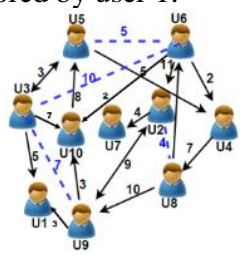

Figure 1. Sample of relations in implicit networks

\subsection{Network 2: Co-readership Implicit Social Network}

The co-readership ISN connects users who bookmark (and presumably read) papers written by the same authors. If user 1 and user 2 have both bookmarked papers written by user 3 , then user 1 and user 2 are connected using the co-readership ISN. This network structure is useful for users who do not yet have publications and therefore cannot have relations in network 1 . The assumption is that users who bookmark the same paper(s) also have similar interests. The strength of the relationship is measured by the number of authors whose libraries overlap. Figure 1 shows an example of the relationships in this network in blue. For example, user 5 and user 6 are connected because they both bookmarked papers written by the same authors; the number of overlapping author names here is five. We show only a part of the graph, and it includes only one of those five authors (user 4).

\subsection{Network 3: Tag-based Implicit Social Network}

The tag-based ISN connects users if they use the same tags to annotate their bookmarked papers. However, we do not check whether users use the same tags to annotate the same papers. We consider the tag similarity between the entire tag cloud associated with each user. We assume that the more similar tags the users have, the higher the interest similarity. While the previous two networks are based on the papers' metadata, this network is based on user-generated data. To build this network, the tags used to annotate the papers are aggregated for each user. The data is preprocessed to make the tags comparable. We follow the method described in [8] to preprocess the tags. All tags are preprocessed by converting them to lowercase, removing the stop words, and then using the porter stemmer tool to remove any additional letters added to the root word to eliminate the effect of the word variation (e.g., the word "social" could have different variations, such as "socialize", "socialization" or "socializing"). The relations in this network also have strengths. The strength of the relation between two users is measured by the number of tags they share. The assumption is that the more tags two users share, the stronger the relationship is between them.

\section{EXPERIMENT DESIGN AND DATASET}

We have conducted different offline experiments to evaluate the recommendation accuracy. We compared three different recommenders that either use pure social relations in the recommendation or incorporate the social relations in collaborative filtering $(\mathrm{CF})$. In addition, we compared the recommenders using each ISN with the corresponding CF used as a baseline approach. We then compared the best recommender for each ISN to test whether any of proposed ISN's recommendations outperform those of the others.

\subsection{Recommendation Approaches}

To determine the effectiveness of different ISNs as good sources for recommendations, we compared the following various existing social recommendation approaches. These recommendation approaches were applied previously to datasets that have explicit social relations and numeric ratings of items (i.e. rating of items using Likert scale). We applied the same approaches to dataset that has implicit social relations and unary ratings of items (i.e. existence of the paper in the user's library).

\subsubsection{Social Recommender}


The social recommender was proposed by [2]. It simply replaces the anonymous nearest neighbors in the user-based CF with the target user's social friends in the social network. To apply the social recommender to one of the proposed ISNs, we found the social friends of each user and used the data from those friends in the same way that we used anonymous peers in $\mathrm{CF}$ by picking the top $\mathrm{N}$ peers and using their bookmarked papers to find candidate papers to recommend to the user. However, in social recommender we replaced the similarity between users that is used in the predication of the target user's rating for unseen items with the weighted strength between users in ISNs.

\subsubsection{Combined Recommender}

The combined recommender integrates neighbors from conventional user-based CF and the target user's social friends to construct a new nearest neighborhood set for the target user [2]. We then used the data from users in the new combined neighbors in the recommendation following the same way as social recommender.

\subsubsection{Amplified Recommender}

The amplified recommender amplifies the social friends' preferences in CF nearest neighbors [9]. First, the nearest neighborhood peers were identified by $\mathrm{CF}$ top-N technique. Then, if the user's social friends were also in the top-N neighbors, we used an amplifying approach to give the preferences from those social friends more weight in the recommendation process. The amplifying function that we used is the one used in [14], which is given by (1):

$$
\operatorname{Min}\left(S_{i j} \times\left(1+\left(\left(N_{i j} / N_{\text {all }, J}\right)\right), 1\right)\right.
$$

where $\mathrm{j}$ is the target user, $\mathrm{i}$ is one of the user's social friends, $\mathrm{S}_{\mathrm{ij}}$ is the similarity between user (j) and user (i) which is calculated by CF approach using the papers that are co-bookmarked by both users, $\mathrm{N}_{\mathrm{ij}}$ is the number of interaction between the target user $(\mathrm{j})$ and the user's social friend (i), and $\mathrm{N}_{\text {all, }}$ is the total number of interactions between the target user ( $\mathrm{j}$ ) and all of the user's social friends. Because the similarity value cannot be greater than 1 , we chose a minimum value between 1 and the amplified value. The interactions between the target user and one of the user's social friends were based on the type of ISN on which we were trying to apply the approach. For example, if we use the co-readership ISN, the number of interactions equals the number of authors that both users have in common (i.e., the number of authors one or more of whose papers both users bookmark).

\subsection{Dataset}

We collected the data for this study from the CiteULike.org social bookmarking Web site, which allows social features such as connecting users, watching users (like following on Twitter), and sharing references. Users of CiteULike can import scientific reference data from other resources such as PubMed and can assign tags to the bookmarked references for future easy access. Using the snowball method, we crawled the CiteULike Web site, starting with 500 randomly chosen, recently active users whose publications and bookmark data we collected. Then, we branched to collect the users' data for the users who had bookmarked their publications or who had bookmarked the same papers as the initial users. The total number of users in this dataset is 13,189 with average number of 1.52 publications, 98.79 bookmarks and 3.81 tags per user. The total number of publications, bookmarks, and tags are 19,774, 1,323,065, and 3,086,565 respectively.

\section{Experiments and Analysis of Results}

In order to evaluate the relevancy of recommended items using the aforementioned ISNs as recommendation resources, information- retrieval-based evaluation methods are usually used such as precision, recall, and F-measure. We conducted two offline experiments using Nfold cross-validation. It is a random selection technique that selects one fraction of the user's bookmarks of size $(1 / \mathrm{N})$ as a testing set and uses the remaining $(\mathrm{N}-1) / \mathrm{N}$ fractions of the user's bookmarks to train the algorithm's model. This process is repeated $\mathrm{N}$ times, each time with different test and training sets. We then judged the prediction's accuracy by calculating the precision and the recall. Our experiments used fivefold cross validation and three different ranks for precision and recall (top two, top five, and top ten).

The first experiment examined which recommendation algorithm produces the best prediction accuracy for each ISN, while the second experiment compared the prediction accuracy of the recommendation for different ISNs. For each ISN, we compared the performance of the three recommendation algorithms - social recommender, combined recommender, and amplified recommender-using precision and recall prediction accuracy measures at three different ranks $(\mathrm{N}=2,5$, and 10). We compared the accuracy differences of the algorithms at 95 percent significance level using a one-way ANOVA, which tests the null hypothesis that no statistical difference exists between the mean values of precision and the recall of the three recommendation approaches, followed by the Tukey post hoc test to rank the algorithms based on the mean differences at all ranks.

\subsection{Results of Different Recommenders for Each ISN}

\subsubsection{Readership ISN (Reciprocal Relations)}

The results of comparing the prediction accuracy of the three recommenders showed statistically significant differences between the means of the precision values (see Figure 2). However, the recall values were insignificant. By applying one-way ANOVA at $\mathrm{p}<0.05$, we found $\mathrm{F}=5.61$ for $\mathrm{P} @ 2, \mathrm{~F}=3.724$ for $\mathrm{P} @ 5$, and F=9.77 for $\mathrm{P} @ 10$. We then applied the Tukey post hoc test to rank the algorithms based on the mean differences of the precision values. We found that the social recommender outperformed the combined recommender, but the difference between the social and the amplified recommenders was insignificant. This means that the amplified recommender benefited from the data from the social friends that make the differences between the social and the amplified recommenders invisible, in contrast with the combined recommender, which deals with the data from anonymous peers and social peers similarly.

\subsubsection{Readership ISN (Unidirectional Relations)}

Using social relations that relate two users if one user bookmarks a paper written by the other caused the social recommender to perform worst. As Figure 2 shows, the social recommender had the lowest precision and recall values in all ranks. One-way ANOVA showed statistically significant differences in both precision and recall values at $\mathrm{p}<0.05(\mathrm{~F}=25.12$ for $\mathrm{P} @ 2, \mathrm{~F}=27.71$ for $\mathrm{P} @ 5, \mathrm{~F}=29.50$ for $\mathrm{P} @ 10, \mathrm{~F}=14.23$ for $\mathrm{R} @ 2, \mathrm{~F}=14.10$ for $\mathrm{R} @ 5$, and $\mathrm{F}=7.35$ for R@10). The Tukey post hoc tests showed that the combined recommender had the highest precision and recall values, followed by the amplified recommender and the social recommender, whose values for all precision and recall are the lowest. Fusing data from CF in 
combined and amplified recommenders enhanced the recommendation in both recommenders similarly, which means that the social data had no effect in this case.

Figure 2: Prediction accuracy measures for different recommendation approaches in each ISN

\subsubsection{Co-readership ISN}

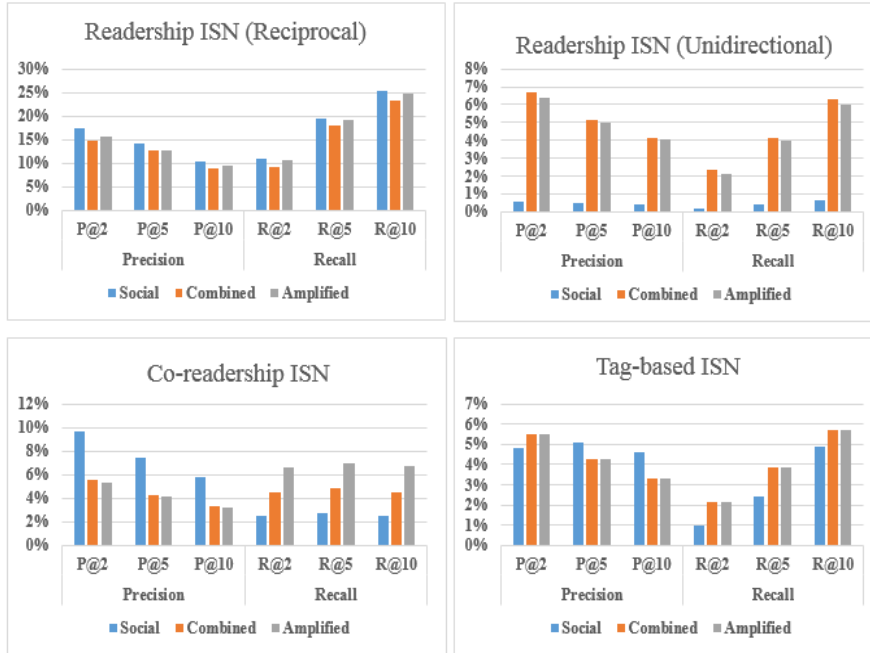

Figure 2 shows that the social recommender outperformed the other two recommenders in precision but not in recall values. To test the significance of the differences, application of one-way ANOVA showed statistical significant differences between the precision values at $\mathrm{p}<0.05(\mathrm{~F}=14.84$ for $\mathrm{P} @ 2, \mathrm{~F}=15.1$ for $\mathrm{P} @ 5$, and $\mathrm{F}=20.13$ for $\mathrm{P} @ 10)$. However, the recall values were insignificant, which means that all the recommenders have similar recall. By applying the $\mathrm{T}$ wetea5wetea5qwqwukey post hoc tests to the precision values for different ranks, we found that the social recommender had the best prediction accuracy, followed by the combined recommender and, finally, the amplified recommender. These results were valid for P@5 and P@10. However, although the precision for the combined and amplified recommenders is similar when recommending two papers, the social recommender has the highest precision value.

\subsubsection{Tag-based ISN}

As Figure 2 shows, the combined and the amplified recommenders displayed almost the same performance for all ranks, which was higher than that of the social recommender with regard to most of the precision and recall values. However, the only significant differences occurred for precision when the top 10 papers were recommended and the recall value as $\mathrm{R} @ 2$. One-way ANOVA showed significant difference of $\mathrm{P} @ 10(\mathrm{~F}=7.46, \mathrm{p}<0.05)$, and the Tukey post hoc test showed that the social recommender outperformed both the combined and the amplified recommenders. One-way ANOVA also showed a significant difference of $\mathrm{R} @ 2(\mathrm{~F}=8.47, \mathrm{p}<0.05)$, and the Tukey post hoc test showed that the combined and amplified recommenders outperformed the social recommender. However, no statistical difference existed between their mean differences. The insignificant results probably occurred because users do not have sufficient number of tags - the average number of tags per user is only 3.81 .

\subsection{Comparing Results with Collaborative Filtering}

In the previous subsection, we compared three recommenders: the social recommender, which uses only the data from social peers, and the combined and amplified recommenders, which incorporate social data into CF. Therefore, we compared the three previous approaches with pure collaborative filtering as a baseline; Table 2 shows the results. We used a T-test to compare each recommender with $\mathrm{CF}$ (significant results are in boldface). The social recommender performs better than $\mathrm{CF}$ in the co-readership ISN and in the readership ISN when the social relations are reciprocal, while CF outperformed the social recommender in readership ISN when the social relations were unidirectional. The results were consistent for all ranks $(\mathrm{N}=2,5,10)$. In the tag-based ISN, the social recommender prediction accuracy was higher than $\mathrm{CF}$ only when ten papers were recommended. The combined recommender also performed better than $\mathrm{CF}$ in some cases (co-readership and unidirectional readership ISNs). In most of the cases for insignificant differences, the prediction accuracy for the three recommenders examined was higher than the CF. That is to say, the recommendation using ISNs as data sources performed better or at least the same as CF.

We gathered information about the performance from the comparisons in Figure 2 and from Table 2. We conclude that the best recommender for each ISN is

- Readership ISN (reciprocal): social recommender

- Readership ISN (unidirectional): combined recommender

- Co-readership ISN: social recommender

- Tag-based ISN: social recommender

\subsection{Which ISN is the Best Recommendation Source?}

We compared the accuracy values of the best performing recommender for each ISN to test, which ISN is the best as a source of recommendation. One-way ANOVA showed significant differences of precision and recall values at $\mathrm{p}<0.05(\mathrm{~F}=81.19$ for $\mathrm{P} @ 2, \mathrm{~F}=123.66$ for P@5, F=139.88 for P@10, F=164.80 for R@2, F=190.91 for $\mathrm{R} @ 5$, and $\mathrm{F}=251.76$ for $\mathrm{R} @ 10$ ). Then, the Tukey post hoc test showed that the readership ISN (reciprocal) has the highest precision performance, followed by the co-readership ISN. Then, the readership ISN (unidirectional), and the tag-based ISN with similar performances. For the recall, the only significant result was that the readership ISN (reciprocal) had the highest recall value.

\begin{tabular}{|c|c|c|c|c|}
\hline & \multirow{2}{*}{ RS } & \multicolumn{3}{|c|}{ Mean value (T-test value and significance level ( $p$-value)) } \\
\hline & & $\mathrm{P@2}$ & P@5 & $\mathrm{P} @ 10$ \\
\hline \multirow{4}{*}{$\begin{array}{l}\text { Readership ISN } \\
\text { (reciprocal) }\end{array}$} & SR & $17.37 \%(t=4.017, \mathrm{p}<0.05)$ & $12.83 \%(\mathrm{t}=4.42, \mathrm{p}<0.05)$ & $9.53 \%(t=3.28, p<0.05)$ \\
\hline & CR & $14.78 \%(t=1.23, \mathrm{p}=0.25)$ & $12.62 \%(t=1.27, \mathrm{p}=0.24)$ & $8.87 \%(\mathrm{t}=0.367, \mathrm{p}=0.84)$ \\
\hline & AR & $15.74 \%(t=2.52, \mathrm{p}<0.05)$ & $12.83 \%(t=1.74, \mathrm{p}=0.12)$ & $9.53 \%(\mathrm{t}=2.063, \mathrm{p}=0.73)$ \\
\hline & $\mathrm{CF}$ & $13.66 \%$ & $11.81 \%$ & $8.79 \%$ \\
\hline \multirow{4}{*}{$\begin{array}{l}\text { Readership ISN } \\
\text { (unidirectional) }\end{array}$} & SR & $0.56 \%(t=-49.64, p<0.05)$ & $0.46 \% \%(t=-55.2, \mathrm{p}<0.05)$ & $0.39 \% \%(t=-79.6, \mathrm{p}<0.05)$ \\
\hline & \begin{tabular}{|l}
$\mathrm{CR}$ \\
\end{tabular} & $6.72 \% \%(\mathrm{t}=2.49, \mathrm{p} \leq 0.05)$ & $5.13 \%(t=1.19, p=0.26)$ & $4.10 \%(t=1.79, p=0.11)$ \\
\hline & AR & $6.39 \%(t=0.17, p=0.871)$ & $5.00 \%(t=-0.47, p=0.64)$ & $4.04 \%(t=0.28, p=078)$ \\
\hline & $\mathrm{CF}$ & $6.39 \%$ & $5.04 \%$ & $4.02 \%$ \\
\hline \multirow{4}{*}{ Co-readership ISN } & SR & $9.72 \%(\mathrm{t}=3.96, \mathrm{p}<0.05)$ & $7.42 \% \%(t=8.39, p<0.05)$ & $5.82 \%(\mathrm{t}=10.26, \mathrm{p}<0.05)$ \\
\hline & CR & $5.56 \%(\mathrm{t}=2.57, \mathrm{p}<0.05)$ & $4.25 \%(t=4.19, \mathrm{p}<0.05)$ & $3.30 \%(t=3.93, \mathrm{p}<0.05)$ \\
\hline & \begin{tabular}{|l|l|} 
AR \\
\end{tabular} & $5.33 \%(t=-0.15, p=0.89)$ & $4.09 \%(\mathrm{t}=0.65, \mathrm{p}=0.53)$ & $3.21 \%(\mathrm{t}=0.89, \mathrm{p}=0.40)$ \\
\hline & $\mathrm{CF}$ & $5.33 \%$ & $4.06 \%$ & $3.17 \%$ \\
\hline \multirow{4}{*}{ Tag-based ISN } & SR & $4.81 \%(t=-0.98, p=0.36)$ & $5.09 \%(t=1.76, p=0.116)$ & $4.60 \%(t=2.73, \mathrm{p} \leqslant 0.05)$ \\
\hline & $\mathrm{CR}$ & $5.47 \%(\mathrm{t}=-1.83, \mathrm{p}=0.10)$ & $4.28 \%(\mathrm{t}=-0.26, \mathrm{p}=0.79)$ & $3.33 \%(t=0.44, \mathrm{p}=0.96)$ \\
\hline & AR & $5.49 \%(t=1.52, p=0.16)$ & $4.25 \%(t=-0.98, p=0.35)$ & $3.32 \%(t=0.36, p=0.73)$ \\
\hline & CF & $5.57 \%$ & $4.28 \%$ & 3.33 \\
\hline
\end{tabular}

\subsection{User Coverage}

While measuring the prediction accuracy of recommendation to filter several recommendation approaches is important, it is not the only way to evaluate the performance of a certain recommendation approach. Non-performance measures, such as serendipity, diversity, novelty, or coverage, can also evaluate recommendation approaches [10]. One 
measure that compares the capability different recommending approaches to produce recommendations for a larger set of users is the coverage measure, which is the ratio of users who receive nonempty recommended sets to the total number of users. The more coverage provided, the better the recommending algorithm.

Because we used social networks to find research papers from the target user's social friends, the user coverage for each recommendation approach using any of the ISNs is the number of users who have social relations using that ISN. We found that the co-readership ISN had the highest user coverage ( 87.25 percent), then tag-based ( 85.55 percent), readership ISN (with unidirectional relations) (37.22 percent), and readership ISN (with reciprocal relations) (1.59 percent). A tradeoff exists between the prediction accuracy and the user coverage: the more accurate the prediction, the smaller the user coverage. Therefore, finding relevant papers using our proposed ISNs is generally beneficial and specifically increases recommendation coverage. We found that in CiteULike dataset that we used in this study, only 18 percent of users have explicitly social friends (i.e., invited), and the average number of social relations per user is only 0.31 .

\section{Conclusions and Future Work}

We proposed three different ISNs based on user bookmarking behavior. We tested three recommendation approaches for each ISN. We then tested them in comparison with CF. We found that in most cases the social recommender produces the best prediction accuracy. We also found that the readership ISN with reciprocal social relations is the best recommendation information resource, followed by the coreadership ISN. However, the co-readership ISN has more user coverage.

In the future, we want to test the proposed implicit social networks with other datasets and/or with different applications to enable us to generalize our findings. We also want to test the recommendations produced by fusing data from explicit and implicit social networks or fusing data from different ISNs. We hypothesize that fusing data from both explicit and implicit social networks can increase the user coverage for the explicit social networks while at the same time increasing the prediction accuracy for the ISNs whose accuracy rates are lower. We also want to test the recommendations produced by ISNs with real users.

\section{References}

[1] Avesani, P., Massa, P., and Tiella, R. A Trust-Enhanced Recommender System Application: Moleskiing. In Proceedings of the 2005 ACM Symposium on Applied Computing, (New York, USA, 2004), ACM Press, 1589-1593.

[2] Bellogín, A., Cantador, I., and Castells, P. A Study of Heterogeneity in Recommendations for a Social Music Service. In Proceedings of the First International Workshop on Information Heterogeneity and Fusion in Recommender Systems, (New York, NY, USA, 2010), ACM, 1-8.

[3] Golbeck, J. (2005). FilmTrust: Movie Recommendations from Semantic Web-Based Social Networks. In ISWC2005 Posters \& Demonstrations, p. PID-72.

[4] Herlocker, J. L., Konstan, J. A. , Terveen, L. G. and Riedl, T. Evaluating collaborative filtering recommender systems, In $A C M$ Trans. Inf. Syst., 22 (2004), 5-53.

[5] Lee, D.H., and Brusilovsky, P. Improving Recommendations Using Watching Networks in a Social Tagging System. In Proceedings of the 2011 iConference, (New York, NY, USA, 2011)), ACM, 33-39.
[6] Lee, D.H., and Brusilovsky, P. Interest Similarity of Group Members: The Case Study of CiteULike. In the WebSci10: Extending the Frontiers of Society On-Line, Raleigh, NC: USA, 2010).

[7] Lee, D. 2013. Personalized Recommendations Based On Users' Information-Centered Social Networks. Doctoral Dissertation. University of Pittsburgh.

[8] Liu, B. Informational Retrieval and Web Search. In Web Data Mining: Exploring Hyperlinks, Contents and Usage Data, (New York, NY, 2007), Springer , 183-236.

[9] Liu, F., and Lee, H.J. Use of Social Network Information to Enhance Collaborative Filtering Performance. Expert Syst. Appl. 37 (2010), 4772-4778.

[10] Ma, H., Yang, H., Lyu, M.R., and King, I. (2008). SoRec: Social Recommendation Using Probabilistic Matrix Factorization. In

Proceedings of the Seventeenth ACM Conference on Information and Knowledge Management, (New York, NY, USA, 2008), ACM, 931940.

[11] Massa, P., and Avesani, P. Trust-Aware Recommender Systems. In Proceedings of the 2007 ACM Conference on Recommender Systems, (New York, NY, USA, 2007), ACM, 17-24.

[12] McNee, S.M., Albert, I., Cosley, D., Gopalkrishnan, P., Lam, S.K., Rashid, A.M., Konstan, J.A., and Riedl, J. On the Recommending of Citations for Research Papers. In Proceedings of the 2002 ACM Conference on Computer Supported Cooperative Work, (New York, NY, USA, 2002), ACM, 116-125.

[13] O'Donovan, J., and Smyth, B. Trust in Recommender Systems. In Proceedings of the Tenth International Conference on Intelligent User Interfaces, (New York, NY, USA, 2005), ACM, 167-174.

[14] Pera, M.S., and Ng, Y.-K. A Personalized Recommendation System on Scholarly Publications. In Proceedings of the Twentieth ACM International Conference on Information and Knowledge Management, (New York, NY, USA, 2011), ACM, 2133-2136.

[15] Pera, M.S., and Ng, Y.-K. Exploiting the Wisdom of Social Connections to Make Personalized Recommendations on Scholarly Articles. J. Intell. Inf. Syst. 42 (2014), 371-391.

[16] Tang, J., Hu, X., and Liu, H. Social Recommendation: A Review. Soc. Netw. Anal. Min. 3 (2013), 1113-1133.

[17] Torres, R., McNee, S.M., Abel, M., Konstan, J.A., and Riedl, J. Enhancing Digital Libraries with TechLens+. In Proceedings of the Fourth ACM/IEEE-CS Joint Conference on Digital Libraries, (New York, NY, USA, 2004), ACM, 228-236 